\title{
THE ELECTRON SPIN RESONANCE (ESR) SPECTRA OF POTASSIUM-ALUMINA-BORATE GLASS DOPED WITH COPPER IONS
}

\author{
P.S. Shirshnev ${ }^{1}$, R.M. Eremina, ${ }^{2,3}$ I.V. Yatsyk ${ }^{2,3}$, Zh.G. Snezhnaia ${ }^{1}$, D.I. Panov ${ }^{1}$, \\ E.V. Shirshneva-Vaschenko', A.E. Romanov' ${ }^{1}$ and V.E. Bougrov ${ }^{1}$ \\ 1ITMO University, Kronverkskiy prospect 49, Saint-Petersburg, 197101, Russia \\ ${ }^{2}$ Zavoisky Physical-Technical Institute, FRC Kazan Scientific Center of RAS, Sibirskiy tract 10/7, \\ Kazan, 420029, Russia \\ ${ }^{3}$ Kazan (Volga Region) Federal University, Kremlevskaya st. 18, Kazan, 420008, Russia
}

Received: May 12, 2018

\begin{abstract}
We investigate electron spin resonance (ESR), absorption, and photoluminescence spectra of potassium-boron copper-containing glasses. In previous works it was found, that the considered glasses demonstrate a red shift of photoluminescence. In present work, we provide an evidence on the formation in glasses specific copper-containing structures that can be responsible for the red shift of photoluminescence "copper" band.
\end{abstract}

\section{INTRODUCTION}

Glasses with ions and clusters of copper are currently considered as attractive materials for phosphors in white LEDs [1], sensitive media for ultraviolet sensors [2], arc sensors [3], and radiation down-converters. We showed in Ref. [3] that for potassium-boron glasses doped with copper and fluorine ions quantum yield of photoluminescence reaches $50 \%$ or even more. The excitation band for such glasses is $320-400 \mathrm{~nm}$, whereas the emission band has the maximum at $514 \mathrm{~nm}$. We also advanced an idea that these glasses can be used as down-converter media in a solar cells or electric arc sensors.

Comparing the maximum position of the luminescence band related to monovalent copper ions in glass according to the data from Ref. [4] with those for the band maximum given in Ref. [3], one finds that the wavelength of $514 \mathrm{~nm}$ is not quite typical for $\mathrm{Cu}^{+}$luminescent centers, as well as for $\mathrm{Cu}^{+-}$ $\mathrm{Cu}^{+}$dimer centers.
In the present work, we search for other types of copper centers in potassium-boron glasses that are responsible for the observed luminescence and accounting for the red shift of the luminescence band, by exploring electron spin resonance (ESR) technique.

\section{EXPERIMENTAL}

We investigate the potassium-alumina-borate glass with the composition $20 \mathrm{~K}_{2} \mathrm{O}-25 \mathrm{Al}_{2} \mathrm{O}_{3}-50 \mathrm{~B}_{2} \mathrm{O}_{3}-1.5$ $\mathrm{Cu}_{2} \mathrm{O}-0.3 \mathrm{Sb}_{2} \mathrm{O}_{3}-3 \mathrm{NaF}$ plus one weight percent of $\mathrm{C}_{12} \mathrm{H}_{22} \mathrm{O}_{11}$ that is identical to the glass studied in Ref. [3]. The absorption and photoluminescence emission spectra were measured with Avantes Avaspec 2048 setup. ESR measurements in the Xband (at $9.4 \mathrm{GHz}$ ) were carried out with ER 200 SRC Bruker (EMX/plus) spectrometer in the temperature range from 5 to $320 \mathrm{~K}$. ESR measurements in the Q-band (at $34 \mathrm{GHz}$ ) were carried out with Bruker ELEXSYS E580 spectrometer in the tem-

Corresponding author: P. Shirshnev, e-mail: pavel.shirshnev@gmail.com

(c) 2018 Advanced Study Center Co. Ltd. 


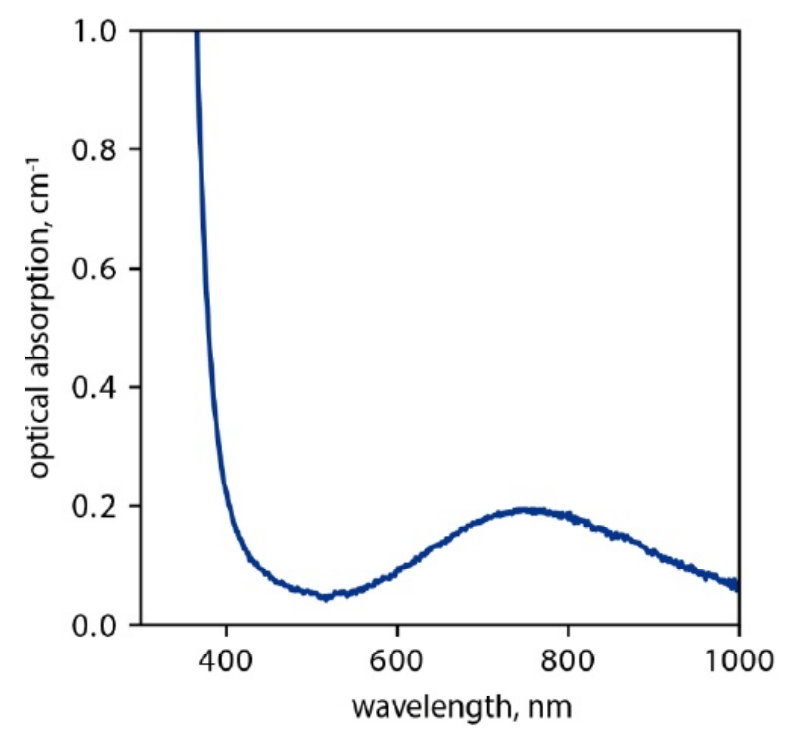

Fig. 1. Optical absorption spectrum of the studied glass.

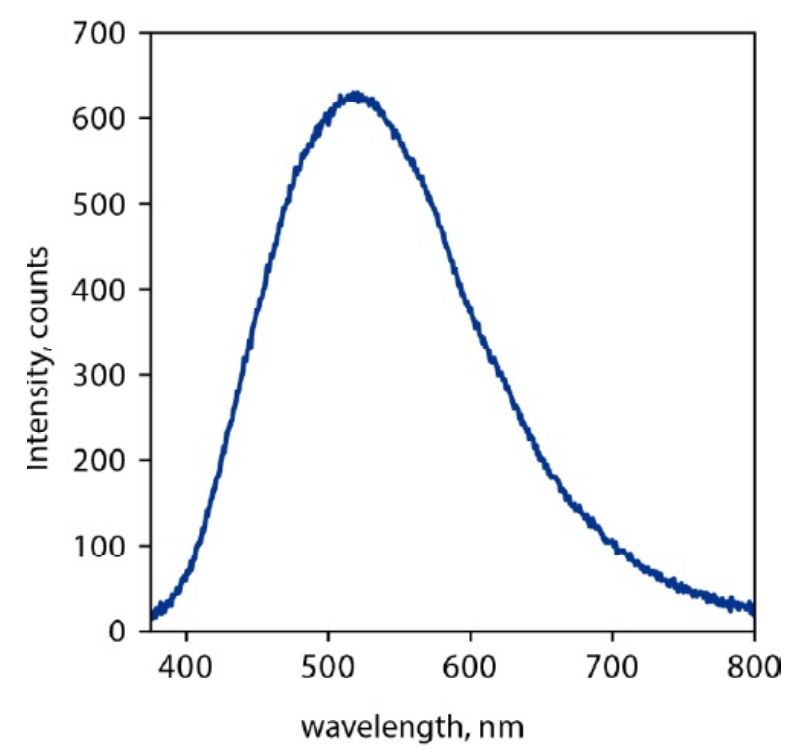

Fig. 2. Photoluminescence emission spectrum upon excitation at $320 \mathrm{~nm}$. The wavelength of luminescence band peak is $514 \mathrm{~nm}$. perature range from 5 to $120 \mathrm{~K}$. The glass samples were fixed in high-purity suprasil quartz-glass tubes by paraffin that allowed to observe spectral lines in a wide temperature range. The ESR measurements cannot recognize nonmagnetic cations $\mathrm{Cu}^{+}$, which can be responsible for luminescence in $490-510 \mathrm{~nm}$ range; however, ESR measurements provide an additional information about other available luminescence copper centers.

\section{RESULTS}

The absorption spectrum of glass under investigation in Fig. 1 shows that there is an absorption band at $740 \mathrm{~nm}$. According to the literature data [6], this band corresponds to the action of divalent copper $\mathrm{Cu}^{2+}$. Considering the concentration of copper oxide (about 1.5\%) in glass with used chemical composition the concentration of divalent copper optical absorption centers turns out to be extremely low with the absorption value of $0.14 \mathrm{~cm}^{-1}$.

Fig. 2 shows the photoluminescence emission band of the glass under the excitation at a wavelength of $320 \mathrm{~nm}$. The band maximum of photoluminescence is $514 \mathrm{~nm}$. Such value of the luminescence band wavelength maximum is intermediate between luminescence caused by monovalent copper ions and copper dimers.

The ESR spectra of the studied glasses are given in Fig. 3 for $7 \mathrm{~K}$ and $115 \mathrm{~K}$ in the Q-band. At lower temperature $(T=7 \mathrm{~K})$ the ESR lineshape can be fitted as the sum of ESR signals of two different type centers. ESR signal of the first type center is fitted by the polycrystalline lineshape with $g_{\|}$and $g_{\perp}=g_{x}=g_{y}$, that is the superposition of Lorentzian lineshape spectra from magnetically anisotropic center with different orientations taking into account

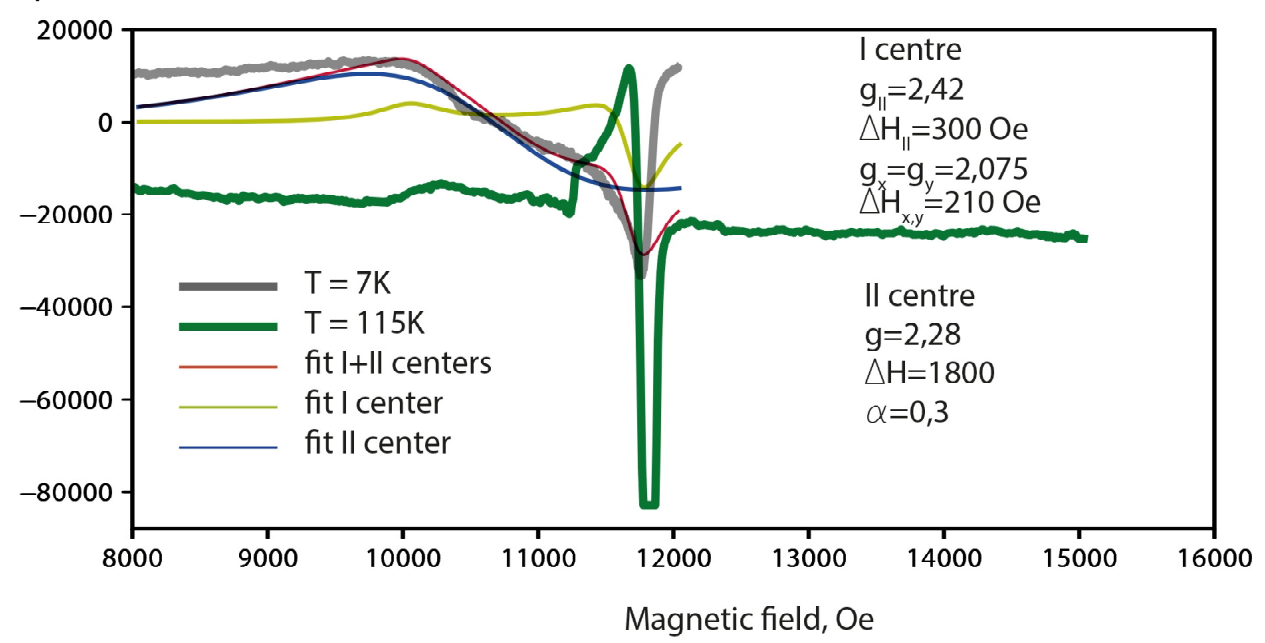

Fig. 3. Electron spin resonance (ESR) spectra of studied glass in the Q-band (at $34 \mathrm{GHz}$ ). Thick lines are the result of experimental measurements; thin lines are the fitting curves. 
their weight factor [6]. The fitting parameters for the first paramagnetic center are $g_{\|}=2.42, \Delta H_{\|}=300 \mathrm{Oe}$ and $g_{\perp}=2.075, \Delta H_{\perp}=210$ Oe. The second type center is isotropic. ESR signal for the second center is fitted by asymmetric Lorentzian lineshape [7], which includes both absorption and dispersion, where a denotes the dispersion-to-absorption $(D / A)$ ratio. The fitting parameters are $g=2.28, \Delta H=1800$ Oe and $\alpha=0.3$. The theoretically calculated ESR lineshape as the sum of the contributions from the first and second type centers is also presented in Fig. 3. As can be seen from Fig. 3, the lineshape and resonance field values for the first center remain almost constant when temperature rises. The g-factor values for the first center are typical for spin $S=1 / 2$ $\mathrm{Cu}^{2+}\left(3 d^{9}\right)$ ions in the solid state, where the orbital moment is quenched by the crystal field [8]. We believe that the manifestation of the second type centers is caused by exchange coupling of spins. For further studies of the second type centers one needs to conduct temperature dependence measurements in the $\mathrm{W}$-band (at $94 \mathrm{GHz}$ ).

Thus, according to the ESR data, there are two types of centers that are related to copper and being responsible for luminescence properties of the studied glasses. One of these centers is the $\mathrm{Cu}^{2+}$ ions, which do not contribute to the observed luminescence, since they have absorption band in the red region of the spectrum. Additional experiments including W-band (at $94 \mathrm{GHz}$ ) ESR measurements are required to understand completely the nature of the second type luminescence copper centers in potassium-alumina-borate glasses.

\section{CONCLUSIONS}

The experimental data obtained support the conclusion that in addition to the main centers in the electron spin resonance range - namely, those related to divalent copper, there are also centers, which, possibly, are responsible for the red shift of the wavelength of photoluminescence band maximum.

\section{ACKNOWLEDGEMENTS}

The ESR spectra of glass were measured within the framework of fundamental research AAAA-A18118030690040-8 at FRC Kazan Scientific Center of RAS. Glass synthesis, optical absorption and photoluminescence spectra were conducted under the support of the Russian Science Foundation, Grant no. 17-72-10216.

\section{REFERENCES}

[1] A. Yasumori, T. Kishi and N. Matsui, US patent (2012) US20120212962A1.

[2] D.S. Agafonova, E.V. Kolobkova, A.I. Ignatiev, N.V. Nikonorov, T.A. Shakhverdov, P.S. Shirshnev, A.I. Sidorov and V.N. Vasiliev // Optical Engineering 54 (2015) 117107.

[3] P.S. Shirshnev, A.E. Romanov, V.E. Bougrov, E.V. Shirshneva-Vaschenko and Z.G. Snezhnaia, In: SPIE Proceedings, Conference 'Photonics for Solar Energy Systems VII' (International Society for Optics and Photonics, 2018), 106881D.

[4] R. Debnath // J. Luminesc. 43 (1989) 375.

[5] P. Shirshnev, V. Tsekhomskiy and N. Nikonorov, Russian Federation Patent RU2466107C2 (2012).

[6] G.M. Zhidomirov, Ya.S. Lebedev, S.N. Dobryakov, N.Ya. Shteinshneider, A.K. Chirkov and V.A. Gubanov, Interpretation of complex ESR spectra (Oxonian Press, New Delhi, India, 1985).

[7] J.P. Joshi and S.V. Bhat // J. Magn. Reson. 168 (2004) 284.

[8] M.A. Fayzullin, R.M. Eremina, M.V. Eremin, A. Dittl, N. van Well, F. Ritter, W. Assmus, J. Deisenhofer, H.-A. Krug von Nidda and A. Loidl // Phys. Rev. B 88 (2013) 174421. 